\title{
Viele Ärzte, freie Entscheidung?
}

Die Befürworter multidisziplinärer Tumorboards bauen auf einen offenen Dialog zwischen Medizinern und Patienten. Das ermögliche eine weniger einseitige Information und eine unabhängige Entscheidung. Aber ist da in der Praxis wirklich etwas dran?

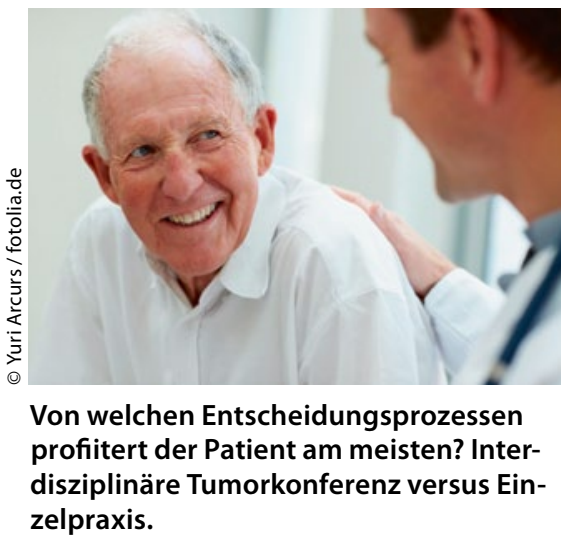

$\mathrm{M}$ oderne Kliniken wie das Nationale Centrum für Tumorerkrankungen in Heidelberg setzen auf multidisziplinäre Zusammenarbeit in der Behandlung ihrer Krebspatienten. Dies soll unter anderem zu einer interaktiven Diskussion führen, wodurch die Voreingenommenheit des einzelnen Arztes weniger ins Gewicht fällt. Aber wirkt es sich tatsächlich positiv aus, wenn etwa Prostatakrebs-Patienten ihre behandelnden Urologen, Radiologen und Onkologen zugleich treffen, statt sie - wie im niedergelassenen Setting üblich - nacheinander zu konsultieren?

Dieser Frage hat sich eine Studie an 701 Patienten mit niedrig-malignem Prostatakarzinom gewidmet, die im Jahr 2009 an drei Therapiezentren in Boston behandelt wurden. Die Patienten erhielten entweder eine Beratung durch ein Tumorboard an einer multidisziplinären Postatakarzinom-Klinik oder aber wurden von individuellen Ärzten aufeinanderfolgend behandelt. Das primäre Outcome war die Entscheidung der Patienten für eine aktive Überwachung (Active Surveillance).

Die Patienten in den multidisziplinären Kliniken entschieden sich erheblich häufiger für die aktive Überwachung als die in Praxen behandelten Patienten: Dies war bei $43 \%$ versus $22 \%$ der Fall. Zugleich sank der Anteil der Männer, die eine Prostatektomie oder eine Be- strahlung erhielten, um etwa $30 \%$ (p < 0,001). Signifikant assoziiert mit der Wahl einer aktiven Überwachung waren ein höheres Alter (Odds Ratio [OR] 1,09), nicht verheiratet zu sein (OR 1,66), ein höherer Charlson-Komorbiditätsindex (OR 1,37) sowie weniger positive Biopsie-Stanzen (OR 0,92). Der stärkste Faktor aber war die Konsultation einer multidisziplinären Klinik (OR 2,15).

Fazit: An multidisziplinär arbeitenden Zentren entscheiden sich Männer mit niedrig-malignem Prostatakarzinom signifikant häufiger für eine Aktive Überwachung statt für eine Prostatektomie oder Bestrahlung. Frühere Studien hatten bereits gezeigt, dass Ärzte vor allem jene Behandlungsmodalität empfehlen, die sie selbst anbieten. Diese Art der Beeinflussung könnte eine Behandlung in einem multidisziplinären Zentrum offenbar tatsächlich verhindern. Gleichwohl müssen Sicherheit und Wirksamkeit der aktiven Überwachung erst noch bewiesen werden.

Christina Berndt

Aizer AA et al. Multidisciplinary care and pursuit of active surveillance in low-risk prostate cancer. J Clin Oncol. 2012;30(25):3071-6.
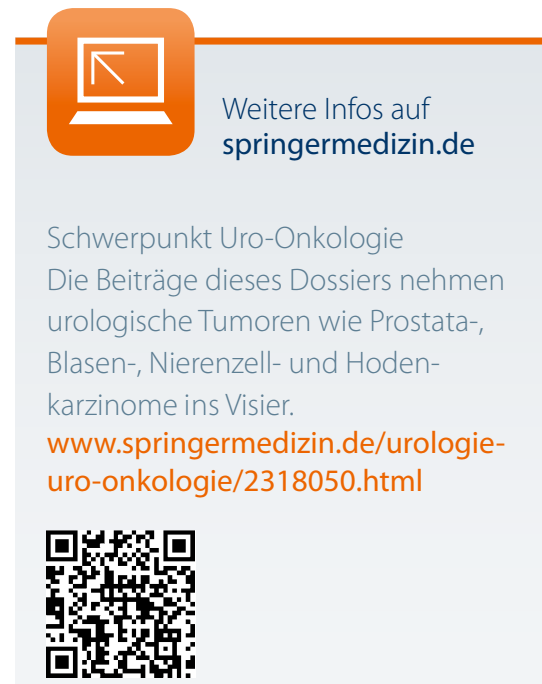